\title{
Metabolic reconfiguration is a regulated response to oxidative stress Chris M Grant
}

Address: Faculty of Life Sciences, The University of Manchester, Oxford Road, Manchester M13 9PT, UK. Email: chris.grant@manchester.ac.uk

Published: 25 January 2008

Journal of Biology 2008, 7:1 (doi: 10.1 I86/jbiol63)

The electronic version of this article is the complete one and can be

found online at http://jbiol.com/content/7/I/I

(C) 2008 BioMed Central Ltd

\begin{abstract}
A new study reveals that, in response to oxidative stress, organisms can redirect their metabolic flux from glycolysis to the pentose phosphate pathway, the pathway that provides the reducing power for the main cellular redox systems. This ability is conserved between yeast and animals, showing its importance in the adaptation to oxidative stress.
\end{abstract}

Organisms are exposed to reactive oxygen species (ROS), such as hydrogen peroxide and the superoxide anion, during the course of normal aerobic metabolism or following exposure to radical-generating compounds. ROS cause wide-ranging damage to macromolecules, which can eventually lead to cell death and thus to aging and a range of diseases [1]. To protect themselves against this damage, cells have effective defense mechanisms, including antioxidant enzymes and free radical scavengers [2]. It is now well established that most cells can adapt to oxidative stress by altering global gene-expression patterns, including transcription and translation of genes encoding antioxidants and other metabolic enzymes. It is becoming increasingly recognized, however, that post-translational changes are key regulators of stress responses. A recent study in Journal of Biology [3] shows that dynamic rerouting of the metabolic flux to the pentose phosphate pathway, with the concomitant generation of the reduced electron carrier nicotinamide adenine dinucleotide phosphate (NADPH), is a conserved post-translational response to oxidative stress.

The pentose phosphate pathway is the source of cellular reducing power in the form of NADPH. NADPH is particularly important during exposure to oxidants because it provides the reducing potential for most antioxidant and redox regulatory enzymes, including the glutathione/ glutaredoxin and thioredoxin systems [4], which are the major systems controlling cellular redox homeostasis. The pentose phosphate pathway is also directly connected to glycolysis, as glucose 6-phosphate is an intermediate in both pathways. Any condition that influences glycolytic activity can thus potentially alter the flux of glucose equivalents through the pentose phosphate pathway, leading to a change in the amount of NADPH generated (Figure 1).

There is increasing evidence that post-translational modification of enzymes, causing rapid and reversible changes in enzyme activity, is a common response to oxidative stress [5]. For example, glyceraldehyde 3-phosphate dehydrogenase (GAPDH) has been identified as a target of oxidative modification in many different cellular systems; it may have a regulatory role as a sensor of oxidative stress conditions [6]. Now, Krobitsch and colleagues [3] provide the first direct evidence that oxidative inhibition of glycolytic enzymes, including GAPDH, is a controlled response that enables cells to redirect their carbohydrate flux from glycolysis to the pentose phosphate pathway, generating $\mathrm{NADPH}$. 


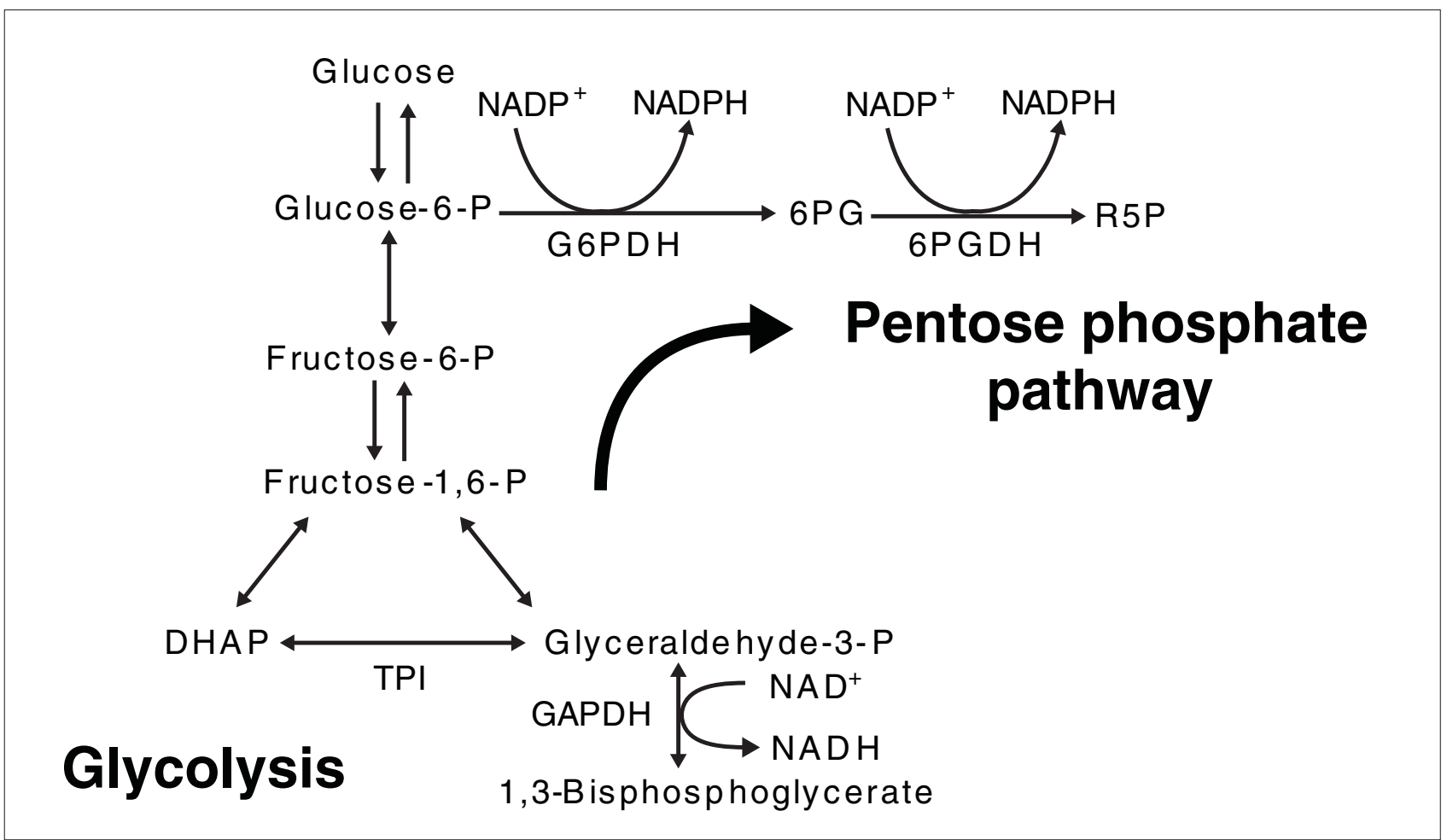

Figure I

A simplified diagram of the link between glycolysis and the pentose phosphate pathway. The pentose phosphate pathway is linked to glycolysis through glucose 6-phosphate; if it is oxidized, it enters the pentose phosphate pathway, whereas if it is isomerized to fructose-6-phosphate, it continues through glycolysis. Inhibiting glycolysis through alterations in the activity of TPI or GAPDH redirects the metabolic flux towards the pentose phosphate pathway and generation of NADPH. Abbreviations: 6PG, 6-phosphogluconate; 6PGDH, 6-phosphogluconate dehydrogenase; DHAP, dihydroxyacetone phosphate; G6PDH, glucose 6-phosphate dehydrogenase; GAPDH, glyceraldehyde 3-phosphate dehydrogenase; P, phosphate; R5P, ribulose 5-phosphate; TPI, triosephosphate isomerase.

The starting point for the study [3] was the previous observation [7] that a decrease in the activity of the glycolytic enzyme triosephosphate isomerase (TPI) confers resistance against oxidative stress conditions caused by the thiol oxidant diamide. Diamide is a membrane permeable, thiol-specific oxidant that promotes the formation of disulphides. It reacts rapidly and spontaneously with glutathione to cause oxidative stress. This finding was extended [3] to show a remarkable correlation between TPI expression levels and oxidant tolerance in both a single-celled eukaryote (the yeast Saccharomyces cerevisiae) and a multicellular animal (the nematode Caenorhabditis elegans). The power of the yeast genetic system was used to test the hypothesis that inactivating TPI blocks glycolysis and results in generation of NADPH from the pentose phosphate pathway. Mutation of the enzyme that performs the first and rate-limiting step in the yeast pentose phosphate pathway (glucose 6-phosphate dehydrogenase, G6PDH) removed the resistance to oxidants, confirming the role of the pentose phosphate pathway in the TPI-dependent oxidant tolerance mechanism. The authors then took the enzyme guanosine diphosphatase (Gdp1p), which oxidizes $\mathrm{NADPH}$ to $\mathrm{NADP}^{+}$, from another yeast (Kluyveromyces lactis). This enzyme is not found in S. cerevisiae and provided a powerful tool to show that altering this redox balance to a more oxidized state causes sensitivity to oxidative stress. Expressing K. lactis Gdp1p in S. cerevisiae also impaired the oxidant tolerance caused by reduced TPI activity; this implied a requirement for NADPH. The definitive evidence of a role for NADPH was provided by measurements of the $\mathrm{NADPH} / \mathrm{NADP}^{+}$ratio, which showed that reducing TPI activity shifts the redox ratio towards a more reducing state; this state is important for maintaining antioxidant activity. Ralser et al. [3] went further by confirming that inactivation of GAPDH functions as a cellular switch for redirecting carbohydrate flux to the generation of NADPH. This is important physiologically because, although oxidative inactivation of GAPDH has been described in many diverse cell types, its exact metabolic consequences have remained poorly defined [8-10]. 
An important insight from the study [3] is the key role played by the pentose phosphate pathway during oxidative stress conditions. G6PDH and 6-phosphogluconate dehydrogenase (6PGDH) catalyze the first two steps of the pentose phosphate pathway. G6PDH catalyzes the key NADPH-production step and is known to have a role in protection against oxidative stress $[11,12]$. Confirming this role, G6PDH and 6PGDH enzyme activities have been shown to be maintained in yeast cells during oxidant exposure $[13,14]$. Direct evidence that flux through the pentose pathway is increased during oxidative stress conditions and, importantly, that NADPH generation via G6PDH and 6PGDH is also increased has so far been lacking. Ralser et al. [3] used a quantitative metabolomic analysis (using liquid chromatography and tandem mass spectrometry) to show that inactivation of TPI results in increased concentrations of pentose phosphate pathway metabolites. Importantly, they found that the more that TPI was inhibited, the more the level of phosphate pathway metabolites increased. One of their key findings is the confirmation that hydrogen peroxide inactivates GAPDH and reroutes metabolic flux into the pentose phosphate pathway, and that this is a way in which the cell balances the cellular reducing environment during exposure to ROS.

This study [3] is one of the first to develop a mathematical model that describes the observed experimental changes in metabolic flux. The model correctly corroborated the experimental findings that reduced TPI or GAPDH activity redirects glucose to the pentose phosphate pathway and thus shifts the NADPH/NADP ${ }^{+}$ratio to a more reduced state. The challenge will now be to extend these systemslevel approaches to integrate further carbohydrate metabolic pathways and the stress conditions that are found in more complicated cellular systems. The increased knowledge of metabolic regulation that is likely to come from these types of study will probably bring about a step change in our understanding of metabolism and might identify novel targets for therapeutic intervention.

In addition, this work [3] could have important implications for our understanding of the metabolic changes that occur during aging. Oxidative damage has often been implicated as a key factor affecting the lifespan of organisms, so metabolic control might have an important role in the aging process. Calorie restriction is the only known nongenetic intervention that extends lifespan in diverse cell types. Studies in yeast cells have shown that altered carbohydrate metabolism fluxes are important in extending lifespan during calorie restriction [15]. Whether regulating the carbohydrate flux through glycolysis and the pentose phosphate pathway has any role in the aging process is unclear at present. It is likely to have a role, however, given that Ralser et al. [3] show that there is a complicated relationship between the requirement for these pathways and the regulation of lifespan in eukaryotic organisms. What is clear is that mutations inactivating these pathways can have a detrimental effect on normal lifespan in both yeast and C. elegans. The work of Krobitsch and colleagues [3] adds to the growing body of literature that links redox regulation and the $\mathrm{NADPH} / \mathrm{NADP}^{+}$ratio with a range of cellular processes, including senescence [16]. Further investigations will be required to elucidate these complex relationships more fully.

The Ralser et al. study [3] demonstrates the need to integrate genomic, biochemical and in silico modeling approaches to understand fully how cells regulate metabolic fluxes during oxidative stress conditions. These types of study are likely to provide new insights into how cells coordinate their metabolic pathways to meet their differing needs during the varied growth and stress conditions to which all cells can be exposed.

\section{References}

I. Gutteridge JM, Halliwell B: Free radicals and antioxidants in the year 2000. A historical look to the future. Ann N Y Acad Sci 2000, 899: 136-147.

2. Temple MD, Perrone GG, Dawes IW: Complex cellular responses to reactive oxygen species. Trends Cell Biol 2005, I5:319-326.

3. Ralser M, Walmelink MM, Kowald A, Gerisch B, Heeren G, Struys EA, Klipp E, Jakobs C, Breitenbach M, Lehrach $H$, Krobitsch S: Dynamic rerouting of the carbohydrate flux is key to counteracting oxidative stress. J Biol 2007, 6:10.

4. Holmgren A: Thioredoxin and glutaredoxin systems. J Biol Chem 1989, 264: 13963-13966.

5. Biswas S, Chida AS, Rahman I: Redox modifications of proteinthiols: emerging roles in cell signaling. Biochem Pharmacol 2006, 7I:55I-564.

6. Chuang DM, Hough C, Senatorov V: Glyceraldehyde-3-phosphate dehydrogenase, apoptosis, and neurodegenerative diseases. Annu Rev Pharmacol Toxicol 2005, 45:269-290.

7. Ralser M, Heeren $G$, Breitenbach $M$, Lehrach $H$, Krobitsch S: Triose phosphate isomerase deficiency is caused by altered dimerization - not catalytic inactivity - of the mutant enzymes. PLOS ONE 2006, I:e30.

8. Schuppe-Koistinen I, Moldeus P, Bergman T, Cotgreave IA: S-thiolation of human endothelial cell glyceraldehyde-3-phosphate dehydrogenase after hydrogen peroxide treatment. Eur J Biochem |994, 221:1033-1037.

9. Ravichandran V, Seres T, Moriguchi T, Thomas JA, Johnston RB: S-thiolation of glyceraldehyde-3-phosphate dehydrogenase induced by the phagocytosis-associated respiratory burst in blood monocytes. J Biol Chem 1994, 269:25010-25015.

10. Grant CM, Quinn KA, Dawes IW: Differential protein S-thiolation of glyceraldehyde 3-phosphate dehydrogenase isoenzymes influences sensitivity to oxidative stress. Mol Cell Biol 1999, 19:26502656.

II. Kletzien RF, Harris PK, Foellmi LA: Glucose-6-phosphate dehydrogenase: a "housekeeping" enzyme subject to tissue-specific regulation by hormones, nutrients, and oxidant stress. FASEB J 1994 , 8:174-|8I.

12. Slekar KH, Kosman DJ, Culotta VC: The yeast copper/zinc superoxide dismutase and the pentose phosphate pathway play overlapping roles in oxidative stress protection. J Biol Chem 1996, 27I:2883 I-28836 
13. Izawa S, Maeda K, Miki T, Mano J, Inoue Y, Kimura A: Importance of glucose-6-phosphate dehydrogenase in the adaptive response to hydrogen peroxide in Saccharomyces cerevisiae. Biochem J 1998, 330:8II-817.

14. Shenton D, Grant CM: Protein S-thiolation targets glycolysis and protein synthesis in response to oxidative stress in the yeast Saccharomyces cerevisiae. Biochem J 2003, 374:5I3-5I 9.

15. Bishop NA, Guarente L: Genetic links between diet and lifespan: shared mechanisms from yeast to humans. Nat Rev Genet 2007, 8:835-844.

16. Ho HY, Cheng ML, Chiu DT: Glucose-6-phosphate dehydrogenase - from oxidative stress to cellular functions and degenerative diseases. Redox Rep 2007, 12:109-118. 